\title{
Thermal Diffusion Analysis by Using Dual Horizon Peridynamics
}

\author{
Bingquan Wang, Selda Oterkus and Erkan Oterkus* \\ Peridynamics Research Centre, Department of Naval Architecture, Ocean and Marine \\ Engineering, University of Strathclyde, Glasgow United Kingdom
}

\section{Funding}

This material is based upon work supported by the Air Force Office of Scientific Research under award number FA9550-18-1-7004. 


\title{
Thermal Diffusion Analysis by Using Dual Horizon Peridynamics
}

\begin{abstract}
In this study, Dual Horizon Peridynamics formulation is presented for thermal diffusion analysis. Lagrangian formalism is utilised to derive the governing equations. The proposed formulation allows utilisation of variable discretisation and horizon sizes inside the solution domain which can result in significant benefits in terms of computational time. To demonstrate the capability of the Dual Horizon Peridynamics formulation, three different example problems are considered including a square plate with temperature and no flux boundary conditions, a square plate under thermal shock loading, and a square plate with an insulated crack. For all problems that are considered good agreement is obtained between peridynamics (PD) predictions and finite element method (FEM) results.
\end{abstract}

Keywords: Thermal diffusion; Dual Horizon; Peridynamics; Non-local

\section{Introduction}

Peridynamics (PD) was introduced by Silling [1] to overcome the limitations of Classical Continuum Mechanics (CCM). Governing equations of PD are in the form of integrodifferential equations rather than partial differential equations as in CCM. Moreover, PD equations do not contain spatial derivatives. Therefore, they are always valid regardless of discontinuities in displacement field due to cracks, etc. Moreover, PD is a non-local continuum mechanics formulation where material points located inside an influence domain, called horizon, can interact with each other. Therefore, it is possible to model problems which show non-classical behaviour including size effects, etc. Although initial applications of PD were limited to structural analysis, original PD formulation was extended to other fields including thermal diffusion [2], porous flow [3], moisture diffusion [4], etc. This study mainly focuses on the application of PD for thermal diffusion analysis.

Nonlocal behaviour can show itself at low temperatures in cryogenic systems. Moreover, nonlocality should also be taken into account when steep temperature gradients exist. In addition, as the devices are becoming smaller, non-local behaviour can emerge at micro- and nano-scales. Therefore, utilisation of non-local formulations such as peridynamics become essential to accurately represent the physics of the problem. There are various studies in the literature focused on peridynamic analysis of thermal diffusion. Amongst these Gerstle et. al. [5] developed a 1-Dimensional multiphysics model coupling thermal diffusion, electrical, mechanical and vacancy diffusion fields to investigate the electromigration phenomenon. Bobaru and Duangpanya [6] introduced a multidimensional bond-based peridynamic formulation for transient heat transfer analysis. Oterkus et. al. [7] developed ordinary statebased peridnamic heat conduction equation based on Lagrangian formalism. To analyse failure prediction in electronic packages, Oterkus et. al. [8] proposed a coupled hygro-thermomechanical model. Xue et. al. [9] developed state-based peridynamic formulation for heat transfer analysis by utilising the domain decomposition method. Wang et. al. [10] utilised Green's function method to develop peridynamic diffusion model. Liao et. al. [11] performed 
peridynamic simulations for heat conduction analysis of functionally gradient materials by considering cracks.

Closed-form solutions of PD equations are generally not available and meshless method is usually utilised to obtain a numerical solution [12]. For spatial discretisation, uniform discretisation scheme is widely used. However, for some problems, using uniform discretisation can unnecessarily increase the computational time since only some part of the solution domain can be solved by using fine discretisation whereas other parts can be modelled by using coarse discretisation. Moreover, variable horizon size can also be required to reduce the computational time or due to the nature of the problem. To overcome these concerns, Dual Horizon Peridynamics was developed by Ren et. al. $[13,14]$ which allows both non-uniform discretisation and variable horizon for mechanical analysis. However, Dual Horizon Peridynamics formulation for thermal diffusion is not currently available in the literature. Therefore, this study presents a novel Dual Horizon Peridynamics formulation for thermal diffusion by utilising Lagrangian formalism. To demonstrate the capability of the Dual Horizon Peridynamics formulation, three different example problems are considered including a square plate with temperature and no flux boundary conditions, a square plate under thermal shock loading, and a square plate with an insulated crack.

\section{Peridynamic Thermal Diffusion Formulation}

The governing equation for peridynamic (PD) thermal diffusion can be derived by solving for the Euler-Lagrange equation [7]

$$
\frac{d}{d t}\left(\frac{\partial L}{\partial \dot{\Theta}}\right)-\frac{\partial L}{\partial \Theta}=0
$$

with the definition of Lagrangian, $L$

$$
L=\int_{V} \mathrm{~L} d V
$$

where $\Theta$ is the temperature and $L$ is the Lagrangian density. In peridynamic framework, the Lagrangian density of a peridynamic material point can be defined as

$$
\mathrm{L}=Z+\rho \hat{s} \Theta
$$

in which $Z$ is thermal potential, $\rho$ is density, and $\hat{s}$ is the heat source per unit mass. 


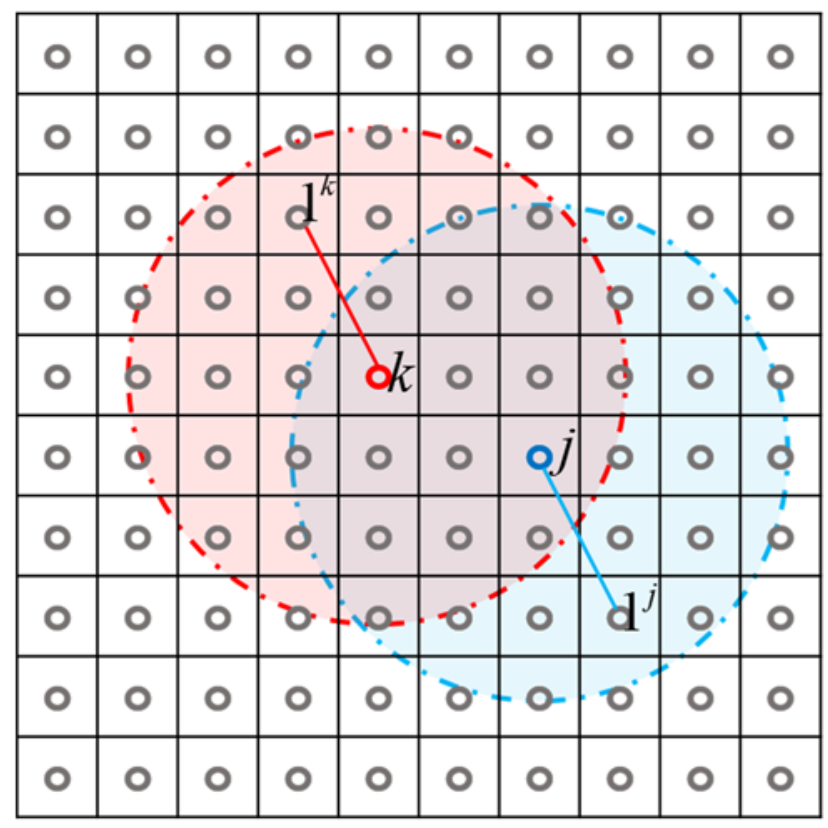

Figure 1. Material points and the horizon

In peridynamics the interactions between material points are nonlocal. Therefore, a material point exchanges heat energy through non-local interactions with its surrounding material points within its horizon, $H$ with a size of, $\boldsymbol{\delta}$ (see Fig.1) [3]. Each material point has a thermal potential which is a function of temperatures of all material points inside its horizon. For a material point $\boldsymbol{x}_{(\boldsymbol{k})}, \boldsymbol{Z}_{(\boldsymbol{k})}$ indicates the thermal potential of this material point. Moreover, due to the heat energy exchange (interaction) between two material points $\boldsymbol{x}_{(\boldsymbol{k})}$ and $\boldsymbol{x}_{(j)}$, two microthermal potential $\boldsymbol{z}_{(\boldsymbol{k})(j)}$ and $\boldsymbol{z}_{(j)(\boldsymbol{k})}$ arise. Microthermal potential, $\boldsymbol{z}_{(\boldsymbol{k})(\boldsymbol{j})}$ depends on the temperature difference between the material point $\boldsymbol{x}_{(\boldsymbol{k})}$ and the material points that it is interacting with inside its horizon. Therefore, the thermal potential $\boldsymbol{Z}_{(\boldsymbol{k})}$ at material point $\boldsymbol{x}_{(\boldsymbol{k})}$ can be defined as

$$
\boldsymbol{Z}_{(\boldsymbol{k})}=\frac{1}{2} \sum_{j=1}^{N_{k}} \frac{1}{2}\left\{\begin{array}{c}
\boldsymbol{z}_{(\boldsymbol{k})(j)}\left[\Theta_{\left(1^{k}\right)}-\Theta_{(k)}, \cdots, \Theta_{\left(N_{k}{ }^{k}\right)}-\Theta_{(k)}\right]+ \\
\boldsymbol{z}_{(j)(\boldsymbol{k})}\left[\Theta_{\left(1^{j}\right)}-\Theta_{(j)}, \cdots, \Theta_{\left(N_{j}\right.}{ }^{j}\right) \\
\left.-\Theta_{(j)}\right]
\end{array}\right\} V_{j}
$$

in which $N_{k}$ and $N_{j}$ are the number of material points inside the horizon of material points $\boldsymbol{x}_{(\boldsymbol{k})}$ and $\boldsymbol{x}_{(j)}$, respectively, $\Theta_{(k)}$ is the temperature of material point $\boldsymbol{x}_{(\boldsymbol{k})}, \Theta_{\left(1^{k}\right)}$ is the temperature of the first material point that interacts with point $\boldsymbol{x}_{(\boldsymbol{k})}$, by the same token for $j$, and $V_{j}$ is the volume associated with the material point $\boldsymbol{x}_{(j)}$. The equation represents that the thermal potential at a material point $\boldsymbol{x}_{(\boldsymbol{k})}$ is the sum of all microthermal potentials associated with that point.

Using the Euler-Lagrange equation given in Eq. (1) for the material point $\boldsymbol{x}_{(\boldsymbol{k})}$ yields the peridynamic governing equation for the thermal diffusion as 


$$
\sum_{j=1}^{N_{k}}\left[-\mathrm{H}_{(k)(j)}+\mathrm{H}_{(j)(k)}\right] V_{(j)}+\rho \hat{s}_{(k)}=0
$$

where

$$
\mathbf{H}_{(k)(j)}=\frac{1}{2} \frac{1}{V_{(j)}}\left[\sum_{i=1}^{N_{k}} \frac{\partial \mathbf{z}_{(\boldsymbol{k})(\boldsymbol{i})}}{\partial\left(\Theta_{(j)}-\Theta_{(k)}\right)} V_{(i)}\right]
$$

and

$$
\mathbf{H}_{(j)(k)}=\frac{1}{2} \frac{1}{V_{(j)}}\left[\sum_{i=1}^{N_{j}} \frac{\partial \mathbf{z}_{(i)(k)}}{\partial\left(\Theta_{(k)}-\Theta_{(j)}\right)} V_{(i)}\right]
$$

The first term in Eq. (5) represents heat flow density from material point $\boldsymbol{x}_{(j)}$ to $\boldsymbol{x}_{(\boldsymbol{k})}$ where second term on the contrary.

The rate of heat energy stored, $\dot{\varepsilon}_{s}$ when heat flow varies over a short period can be expressed as

$$
\dot{\varepsilon}_{s}=C_{v} \frac{\partial \Theta}{\partial t}
$$

where $C_{v}$ is the specific heat capacity. Moreover, the heat source $\hat{s}$ can defined as

$$
\hat{s}=\dot{\varepsilon}_{s}-s_{b}
$$

in which $s_{b}$ is the heat source due to volumetric heat generation per unit mass. Therefore, the peridynamic thermal diffusion equation given in Eq. (5) can rewritten as

$$
\rho C_{v} \dot{\Theta}\left(\boldsymbol{x}_{(\boldsymbol{k})}, t\right)=\sum_{j=1}^{N_{k}}\left[\mathrm{H}_{(k)(j)}-\mathrm{H}_{(j)(k)}\right] V_{(j)}+h_{x}\left(\boldsymbol{x}_{(k)}, t\right)
$$

where heat source due to volumetric heat generation can expressed as

$$
h_{x}\left(\boldsymbol{x}_{(\boldsymbol{k})}, t\right)=\rho s_{b}\left(\boldsymbol{x}_{(\boldsymbol{k})}, t\right)
$$

If bond based peridynamic thermal diffusion model is considered, the heat flow density between two material points is assumed to be a function of the temperature difference between only the interacting material points. Thus,

and

$$
\mathbf{H}_{(k)(j)}=\frac{1}{2} \frac{\partial \mathbf{z}_{(\boldsymbol{k})(j)}}{\partial\left(\Theta_{(j)}-\Theta_{(k)}\right)}
$$




$$
\mathrm{H}_{(j)(k)}=\frac{1}{2} \frac{\partial \mathbf{z}_{(j)(\boldsymbol{k})}}{\partial\left(\Theta_{(k)}-\Theta_{(j)}\right)}
$$

For microthermal potentials,

$$
\boldsymbol{z}_{(k)(j)}=\frac{1}{2} \frac{\mathrm{k}}{2} \frac{\left(\Theta_{(j)}-\Theta_{(k)}\right)^{2}}{\left|\boldsymbol{x}_{(j)}-\boldsymbol{x}_{(k)}\right|}
$$

and

$$
\boldsymbol{z}_{(j)(k)}=\frac{1}{2} \frac{\mathrm{k}}{2} \frac{\left(\Theta_{(k)}-\Theta_{(j)}\right)^{2}}{\left|\boldsymbol{x}_{(k)}-\boldsymbol{x}_{(j)}\right|}
$$

heat flow densities given in Eqs. (12) and (13) can be written as

$$
\begin{aligned}
& \mathrm{F}_{(k)(j)}=\frac{\mathrm{K}}{2} \frac{\Theta_{(j)}-\Theta_{(k)}}{\left|\boldsymbol{x}_{(j)}-\boldsymbol{x}_{(k)}\right|} \\
& \mathrm{F}_{(j)(k)}=\frac{\mathrm{k}}{2} \frac{\Theta_{(k)}-\Theta_{(j)}}{\left|\boldsymbol{x}_{(k)}-\boldsymbol{x}_{(j)}\right|}
\end{aligned}
$$

where PD microconductivity, $\mathrm{K}$ can be expressed for 2-Dimensional solution domains as

$$
\mathrm{x}=\frac{6 k}{\pi h \delta^{3}}
$$

in which $k$ is the heat conductivity, $h$ is the thickness of the geometry and $\delta$ is the horizon size.

\section{Dual Horizon Peridynamics for Thermal Diffusion}

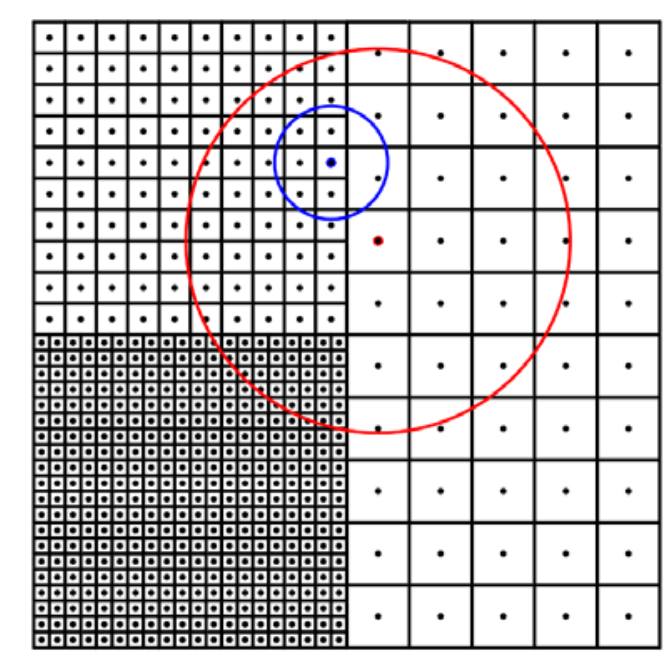

Figure 2. Non-uniform discretization with different horizon sizes. 
The uniform discretization with constant horizon size is commonly used in peridynamic simulations since it is simple to implement for the whole geometry. However, for some problems, using finer discretisation size at all locations inside the solution domain can be computationally time consuming. Thus, it is essential to use finer discretisation size at locations with high temperature gradient and coarse discretisation size can be used elsewhere. Moreover, utilising variable horizon sizes can also be required either due to computational or problem specific reasons.

Note that in Eqs. (12) and (13), the microthermal potential being zero means one of the material point is not within the horizon of the other material point. As shown in Fig. 2, although the material point with a blue (smaller) horizon is inside the horizon of the material point with red (larger) horizon, the material point with a red horizon is outside of the horizon of the material point with a blue horizon. Therefore, the heat flow densities given in Eqs. (16) and (17) between material points $\boldsymbol{x}_{(j)}$ and $\boldsymbol{x}_{(\boldsymbol{k})}$ can be rewritten as

$$
\begin{aligned}
& \mathrm{H}_{(k)(j)}=\alpha_{(k)(j)} \frac{\mathrm{K}_{k}}{2} \frac{\Theta_{(j)}-\Theta_{(k)}}{\left|\boldsymbol{x}_{(j)}-\boldsymbol{x}_{(k)}\right|} \\
& \mathrm{H}_{(j)(k)}=\alpha_{(j)(k)} \frac{\mathrm{K}_{j}}{2} \frac{\Theta_{(k)}-\Theta_{(j)}}{\left|\boldsymbol{x}_{(j)}-\boldsymbol{x}_{(k)}\right|}
\end{aligned}
$$

with

$$
\begin{aligned}
& \alpha_{(k)(j)}=\left\{\begin{array}{l}
1, \mathbf{z}_{(\boldsymbol{k})(j)} \neq 0 \\
0, \mathbf{z}_{(\boldsymbol{k})(j)}=0
\end{array}\right. \\
& \alpha_{(j)(k)}=\left\{\begin{array}{l}
1, \mathbf{z}_{(j)(k)} \neq 0 \\
0, \mathbf{z}_{(j)(k)}=0
\end{array}\right.
\end{aligned}
$$

and

$$
\begin{aligned}
\mathrm{K}_{k} & =\frac{6 k}{\pi h \delta_{k}^{3}} \\
\mathrm{~K}_{j} & =\frac{6 k}{\pi h \delta_{j}^{3}}
\end{aligned}
$$


where $\boldsymbol{\delta}_{\boldsymbol{k}}$ and $\boldsymbol{\delta}_{\boldsymbol{j}}$ are the horizon sizes of the material points $k$ and $j$, respectively.

\section{Results}

To demonstrate the capabilities of the current Dual Horizon Peridynamics thermal diffusion formulation three different problems are considered including a square plate with temperature and no flux boundary conditions, a square plate under thermal shock with insulated boundaries and a square plate with an insulated crack. Uniform or non-uniform discretisation was utilised with constant or variable horizon size. Peridynamic predictions are compared with finite element analysis (FEA) results obtained by using ANSYS, a commercial finite element software.

\subsection{Plate with temperature and no flux boundary conditions}

For the first example problem, an isotropic square plate with dimensions of length $(\mathrm{L})=$ width $(\mathrm{W})=0.01 \mathrm{~m}$ is considered (Fig.3). The plate has a thickness of $h=0.001 \mathrm{~m}$ and is subjected to temperature boundary conditions of $\Theta(x=-0.005, y)=0^{\circ} \mathrm{C}$ and $\Theta(x=0.005, y)=$ $10^{\circ} \mathrm{C}$ at the left and right edges, respectively. The upper and bottom boundaries are insulated boundaries. Boundary conditions are applied by introducing fictitious regions, $R_{c}$ and following the approach described in [7]. Specific heat capacity $C_{v}$, thermal conductivity $k$ and mass density $\rho$ are specified as $64 \mathrm{~J} / \mathrm{kgK}, 233 \mathrm{~W} / \mathrm{mK}$ and $260 \mathrm{~kg} / \mathrm{m}^{3}$, respectively.

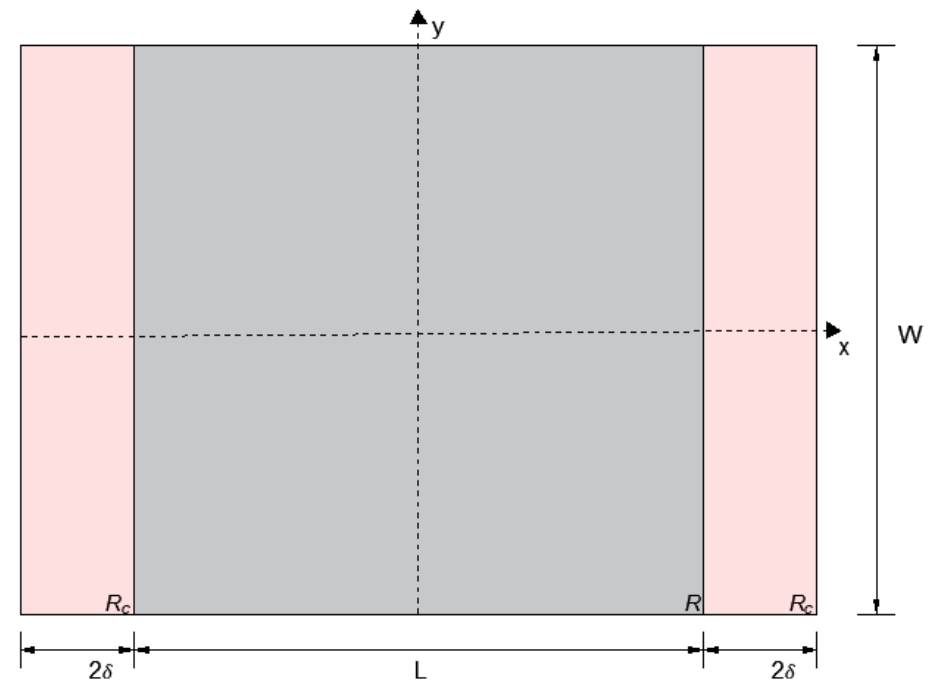

Figure 3. Peridynamic model of the plate

The geometry is discretised in various forms in PD model. The solution domain is split into two equal regions as Region 1 and Region 2 from the vertical axis, $y$. Mesh ratio $\mathrm{k}$ is defined as

$$
\mathrm{k}=\frac{\Delta_{1}}{\Delta_{2}}
$$

where $\Delta_{1}$ indicates the spacing between the material points in Region 1 and $\Delta_{2}$ indicates the spacing between the material points in Region 2 . 
The cases are evaluated with various horizon size combinations and horizon size ratio $(\mathrm{m})$ is defined as

$$
\mathrm{m}=\frac{\delta_{1}}{\delta_{2}}
$$

where $\delta_{1}$ is the horizon size in Region 1 and $\delta_{2}$ is the horizon size in Region 2. The relationship between horizon size and spacing is given as

$$
\delta_{i}=n \Delta_{i}
$$

where $i=1,2$ represents Regions 1 and 2, respectively, whereas $n=1, \ldots, 5$ represents the size the horizon. For example, if $\delta_{2}=3 \Delta_{2}$, the horizon in Region2 has a size of three material points in radius.

\subsubsection{Plate with Uniform Discretisation and Identical Horizon Sizes}

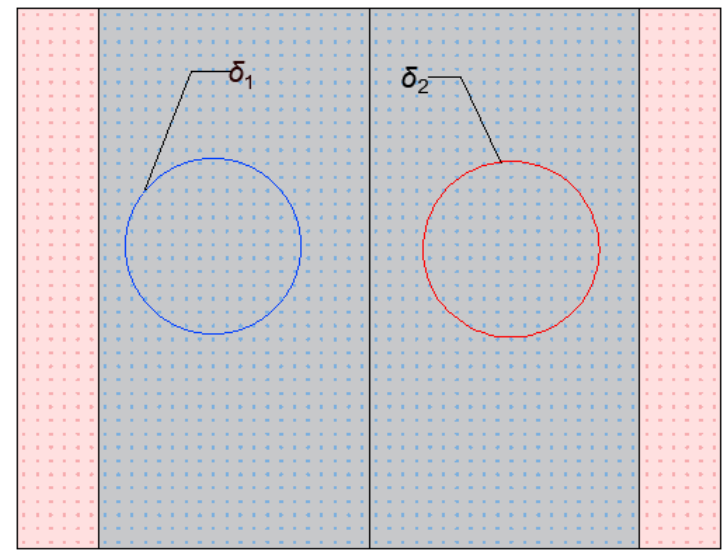

Figure 4. PD model with uniform discretisation and identical horizon sizes

In the first case, as shown in the Fig. 4 , the plate is discretised uniformly with the distance between material points $\Delta_{1}=\Delta_{2}=1.0 \times 10^{-4} \mathrm{~m}$ in horizontal and vertical directions. All material points have an identical horizon size $\left(\delta_{1}=\delta_{2}\right)$. Therefore, $\mathrm{k}$ and $\mathrm{m}$ parameters defined in Eqs. (25) and (26) are both equal to 1. The peridynamic solution of the temperature variations along the horizontal central axis are obtained by using explicit time integration with a time step size of $1.0 \times 10^{-7} \mathrm{~s}$ and compared with the finite element method (FEM) results by using PLANE55 element of ANSYS.

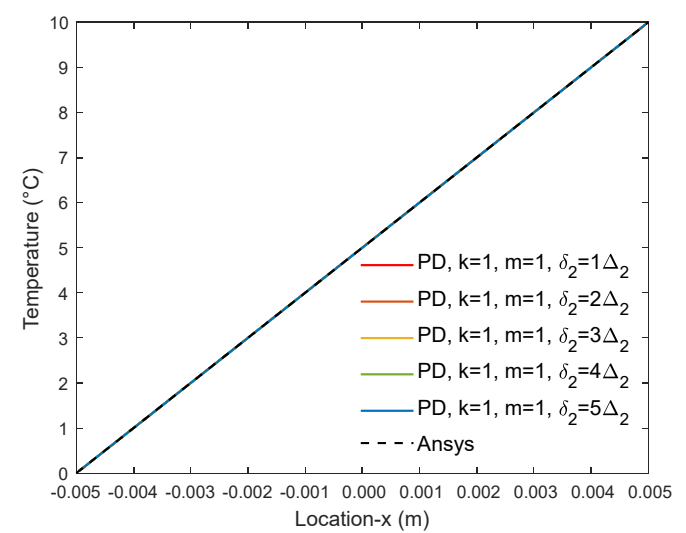

Figure 5. Temperature variations from $P D$ and FEM predictions at $y=0$ when $k=1, m=1$ 
As can be seen in Fig.5, the PD predictions have a good agreement with FEM results for all horizon sizes for uniform discretisation and identical horizon sizes.

\subsubsection{Plate with Uniform Discretisation and Different Horizon Sizes}

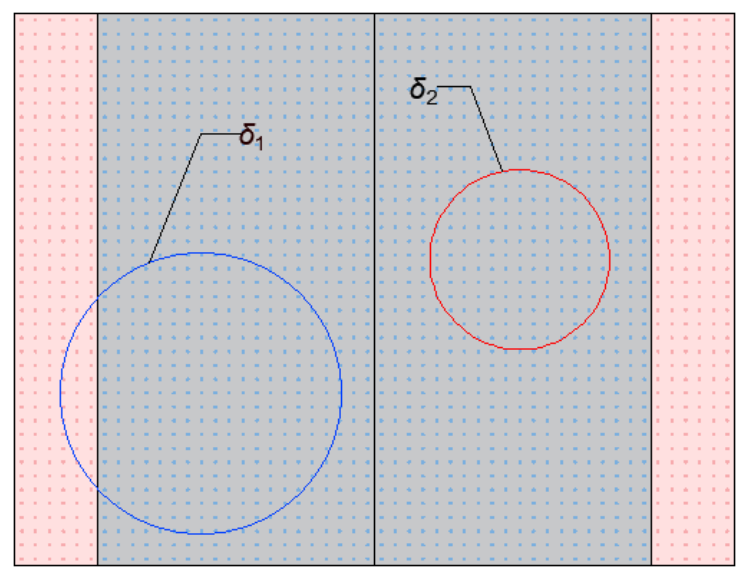

Figure 6. PD model with uniform discretisation and different horizon sizes

In order to investigate the capability of the dual horizon concept, the square plate is discretised with the same discretization parameters $\left(\Delta_{1}=\Delta_{2}=1.0 \times 10^{-4} \mathrm{~m}\right)$ as in the former case. However, the horizon sizes in Regions 1 and 2 of the plate are different (see Fig. 6). The horizon size of the Region 1 is set twice big of the horizon size of Region $2\left(\delta_{1}=2 \delta_{2}\right)$. Therefore, $\mathrm{k}$ and $\mathrm{m}$ parameters are equal to 1 and 2 , respectively.

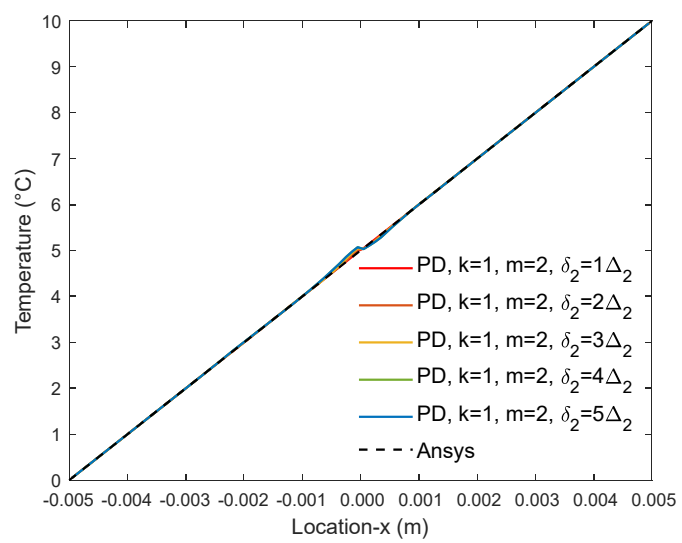

Figure 7. Temperature variations from $\mathrm{PD}$ and FEM predictions at $\mathrm{y}=0$ when $\mathrm{k}=1, \mathrm{~m}=2$

The peridynamic solution of the temperature variations along horizontal central axis are obtained by using explicit time integration with a time step size of $1.0 \times 10^{-7} \mathrm{~s}$ and compared with FEM results as shown in Fig. 7. The PD predictions generally agree well with FEA. However, there is small difference at the interface of Regions 1 and 2 for larger horizon sizes and increases as the horizon size increases. 


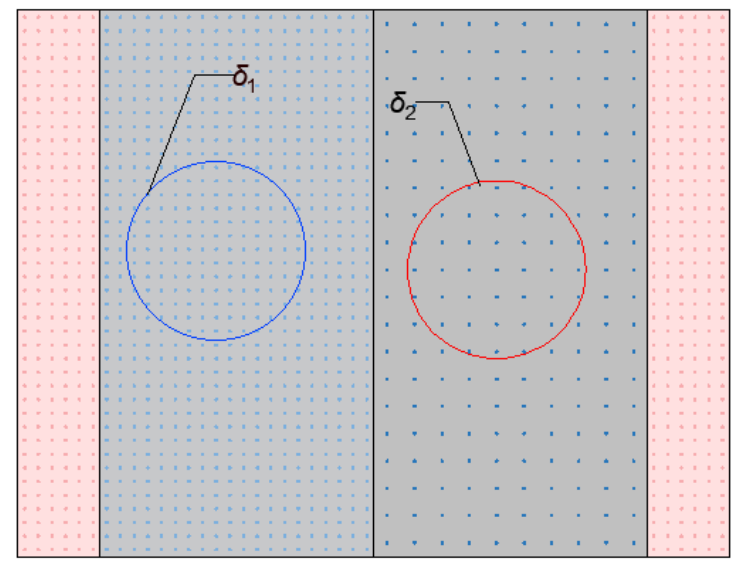

Figure 8. PD model with non-uniform discretisation and identical horizon sizes

In the third case, the square plate has the same geometric parameters, material properties and boundary conditions with the first case. Since refined discretisation increases the computational cost, in order to examine the non-uniform discretisation on heat conduction, the discretisation size in Region 2 is increased to a size of $\Delta_{2}=2.0 \times 10^{-4} \mathrm{~m}$ in horizontal and vertical directions whereas the discretisation size in Region 1 is same as uniform cases which is $\Delta_{1}=$ $1.0 \times 10^{-4} \mathrm{~m}$ (Fig.8). The horizon sizes remain the same in each region as the first uniform case. Therefore, $\mathrm{k}$ and $\mathrm{m}$ parameters are equal to 0.5 and 1 , respectively.

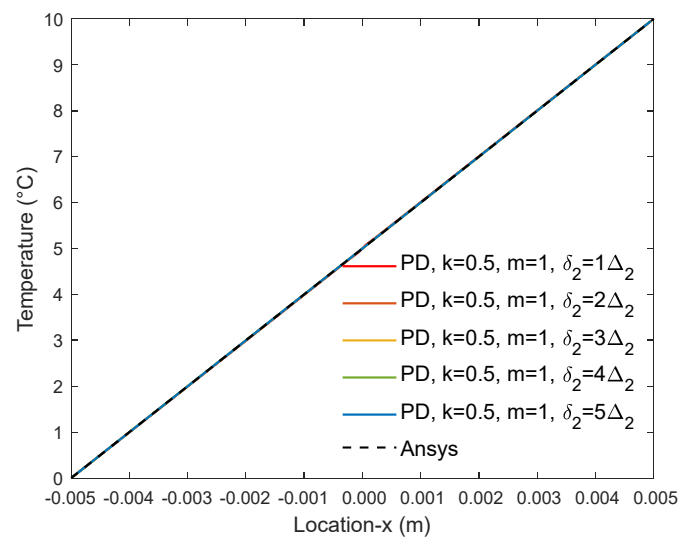

Figure 9. Temperature variations from PD and FEM predictions at $\mathrm{y}=0$ when $\mathrm{k}=0.5, \mathrm{~m}=1$

The peridynamic solution of the temperature variations along the horizontal central axes are obtained by using explicit time integration with a time step size of $1.0 \times 10^{-7} \mathrm{~s}$. As shown in Fig. 9, the PD predictions for the temperature variations agree well with the FEM results for various horizon sizes. 


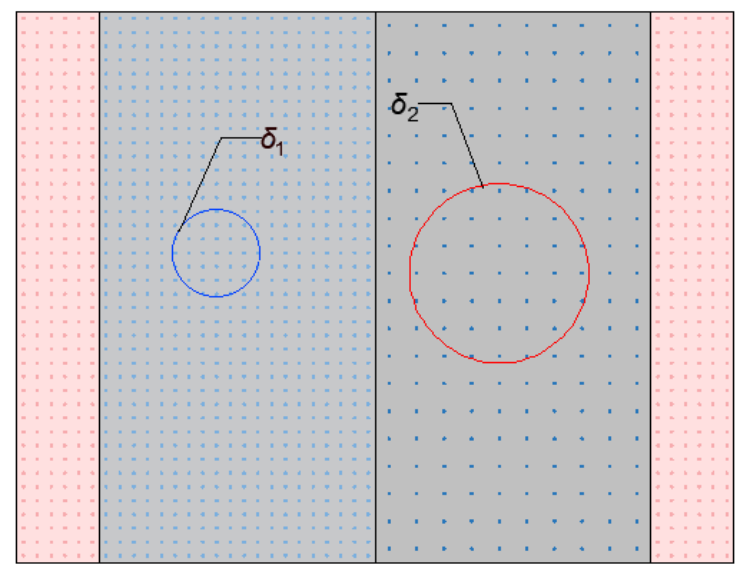

Figure 10. PD model with non-uniform discretisation and different horizon sizes

In peridynamic numerical simulations computational time depends on not only the discretisation size but also the number of material points within its horizon. The third case shows a good agreement of PD and FEM predictions for various horizon sizes with a nonuniform discretisation. Therefore, this last case inherits the non-uniform discretisation from the third case and the horizon side in Region 1 is reduced to half of the horizon size in Region 2, i.e. $\delta_{2}=2 \delta_{1}$ (see Fig. 10). Since $\Delta_{2}=2 \Delta_{1}$ and $\delta_{2}=2 \delta_{1}$, horizon in each region contains same number of material points within their horizon. Moreover, $\mathrm{k}$ and $\mathrm{m}$ parameters are both equal to 0.5 , respectively.

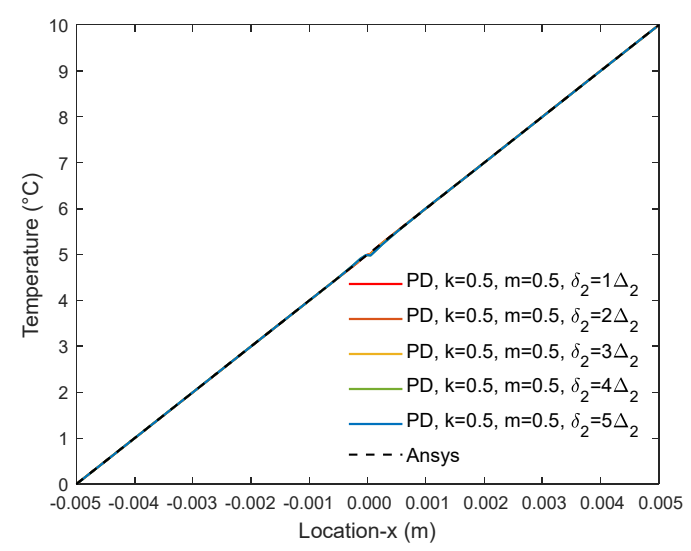

Figure 11. Temperature variations from PD and FEM predictions at $\mathrm{y}=0$ when $\mathrm{k}=0.5$, $\mathrm{m}=0.5$

As observed in the Fig. 11, the PD numerical results mostly agrees well with the FEM results. However, as the horizon size grows, a slight difference is observed at the interface of Regions 1 and 2 . 


\subsection{Plate under thermal shock with insulated boundaries}

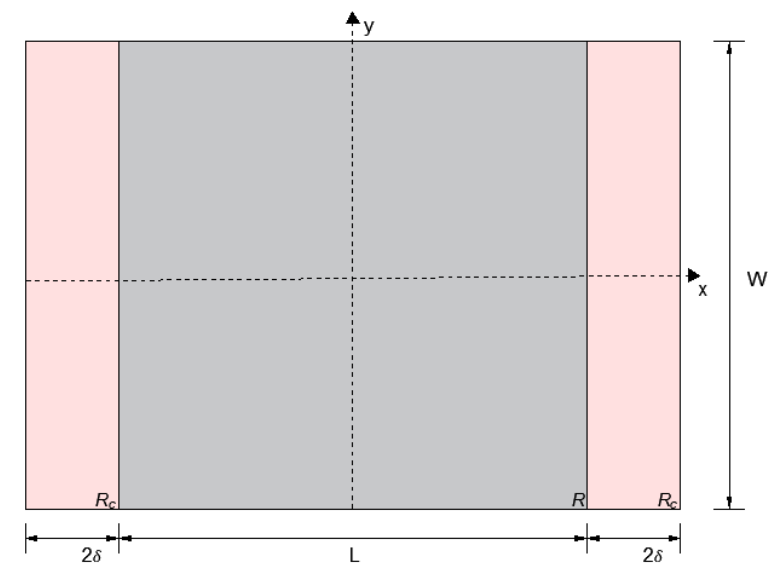

Figure 12. Peridynamic model of the plate under thermal shock loading

In the second example problem, an isotropic square plate with insulated boundaries is subjected to a thermal shock loading on the right edge. The geometric parameters are $10 \mathrm{~m}$ in length (L) and width (W) with a thickness of $(h) 1 \mathrm{~m}$ (see Fig. 12). The specific heat capacity $C_{v}$, thermal conductivity $k$ and mass density $\rho$ are specified as $C_{v}=1 \mathrm{~J} / \mathrm{kgK}, k=1 \mathrm{~W} / \mathrm{mk}$ and $\rho=1 \mathrm{~kg} / \mathrm{m}^{3}$, respectively. The initial condition and boundary conditions are stated as

$$
\Theta(x, y, t=0)=0^{\circ} \mathrm{C}
$$

and

$$
\begin{array}{cc}
\Theta, x(x=0, y)=0^{\circ} \mathrm{C}, & \mathrm{t}>0 \\
\Theta, y(x, y= \pm 5)=0^{\circ} \mathrm{C}, & \mathrm{t}>0 \\
\Theta(x=0, t)=5 t e^{-2 t}, & \mathrm{t}>0
\end{array}
$$

\subsubsection{Plate with Uniform Discretisation and Identical Horizon Sizes}

In the first case, the PD model has a uniform mesh with spacing $\Delta_{1}=\Delta_{2}=0.02 \mathrm{~m}$ and identical horizon sizes in each region $\left(\delta_{1}=\delta_{2}\right)$. Thus, $\mathrm{k}$ and $\mathrm{m}$ parameters are both equal to 1 . The peridynamic solution of the temperature variations along central horizontal axis are obtained by using explicit time integration with a time step size of $5.0 \times 10^{-4} \mathrm{~s}$.

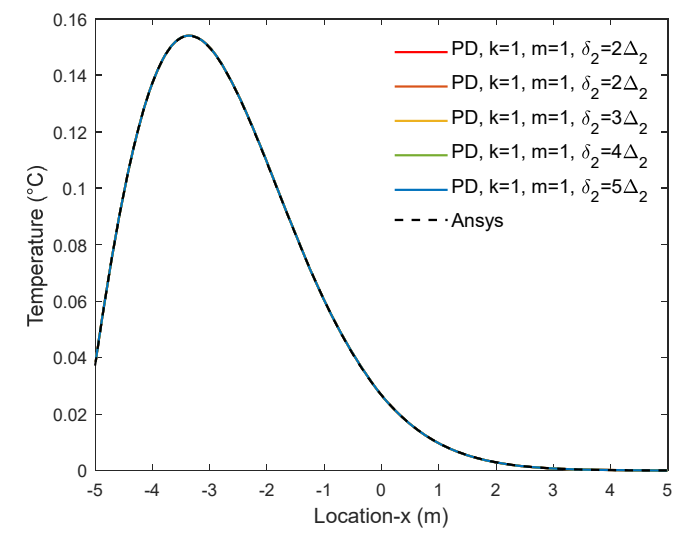

(a)

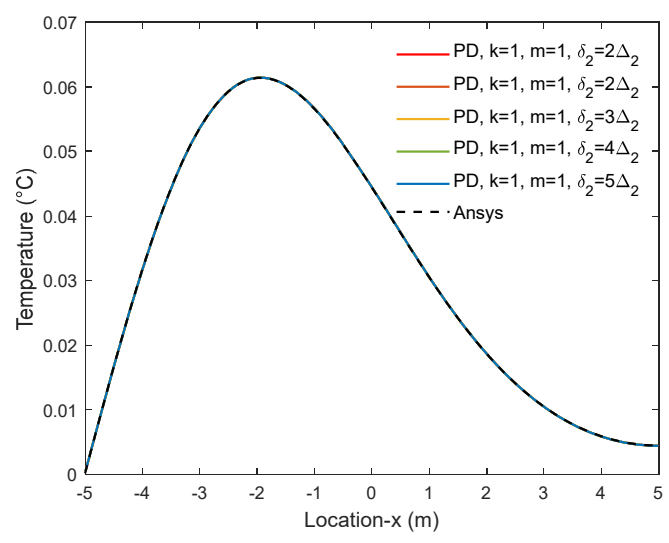

(b)

Figure 13. Temperature variations from $P D$ and FEM predictions when (a) $t=3 s$, (b) $t=6 s$ at $\mathrm{y}=0, \mathrm{k}=1, \mathrm{~m}=1$. 
The results are assessed with various horizons and predicted at time $t=3 \mathrm{~s}$ and $\mathrm{t}=6 \mathrm{~s}$. Both PD and FEM results are shown in Fig. 13. As can be observed, the PD model results agree well with FEM results for various horizons with uniform discretisation.

\subsubsection{Plate with Uniform Discretisation and Different Horizon Sizes}

In the second case, the numerical model has the same mesh configuration with the first case. In order to investigate the capability of the dual horizon concept, horizon size in Region 2 is doubled, i.e. $\delta_{1}=2 \delta_{2}$. Therefore, $\mathrm{k}$ and $\mathrm{m}$ parameters are equal to 1 and 2 , respectively. The second case adopts the identical time step with the former case and the temperature variations along the central horizontal axis are plotted for time $t=3 \mathrm{~s}$ and $\mathrm{t}=6 \mathrm{~s}$ for various horizons and compared with FEM results.

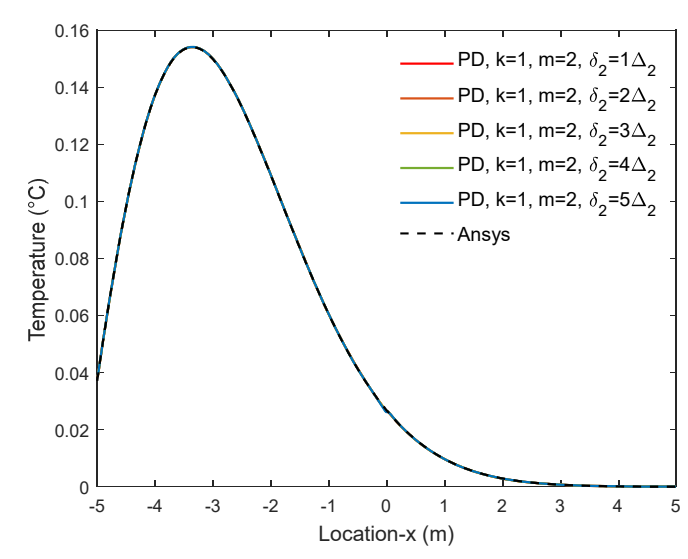

(a)

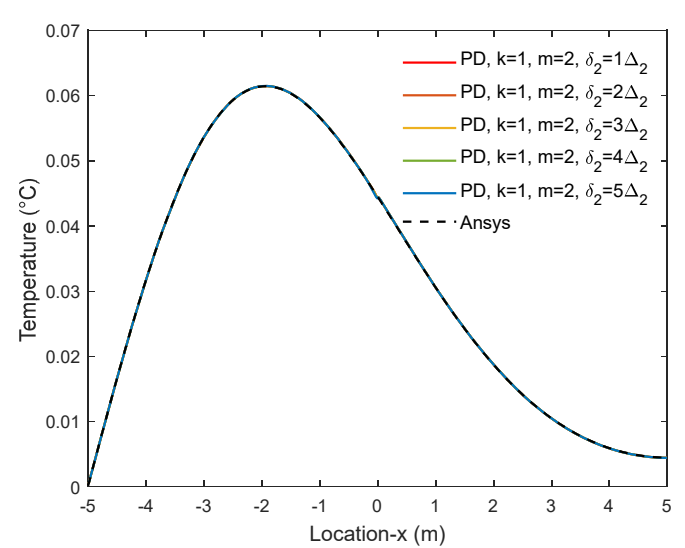

(b)

Figure 14. Temperature variations from PD and FEM predictions when (a) $t=3 s$, (b) $t=6 s$ at $\mathrm{y}=0, \mathrm{k}=1, \mathrm{~m}=2$.

As shown in Fig. 14, PD results for various horizon sizes generally agree well with FEM results. As the horizon size increases, a slight difference is observed at the interface of Regions 1 and 2 at both $t=3 s$ and $t=6 s$. 


\subsubsection{Plate with Non-uniform Discretisation and Identical Horizon Sizes}

In the third case, the geometry is discretized with non-uniform discretisation. The spacing between material points in Region 2 is twice the spacing in Region 1, i.e. $\Delta_{1}=0.02 m, \Delta_{2}=$ $0.04 \mathrm{~m}$. However, the horizon sizes are identical in each regions, i.e. $\delta_{1}=\delta_{2}$. Therefore, $\mathrm{k}$ and $\mathrm{m}$ parameters are equal to 0.5 and 1 , respectively. The peridynamic solution of the temperature variations along the horizontal central axis at times $\mathrm{t}=3 \mathrm{~s}$ and $\mathrm{t}=6 \mathrm{~s}$ are evaluated with explicit time integration with a time step size of $5.0 \times 10^{-4} \mathrm{~s}$ and compared with FEM results.

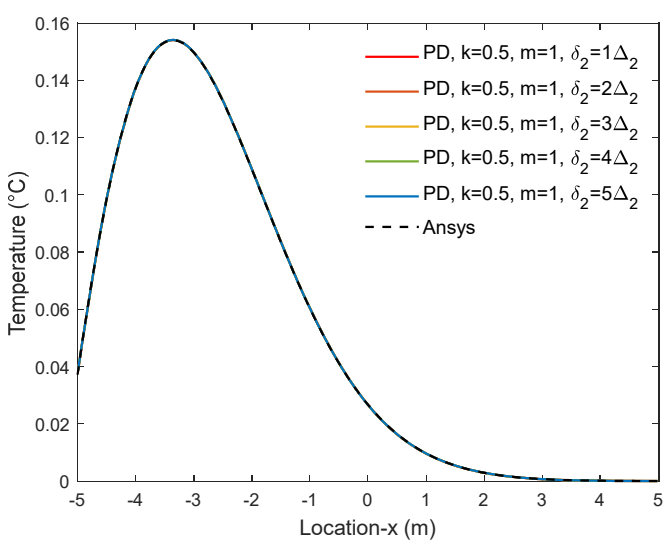

(a)

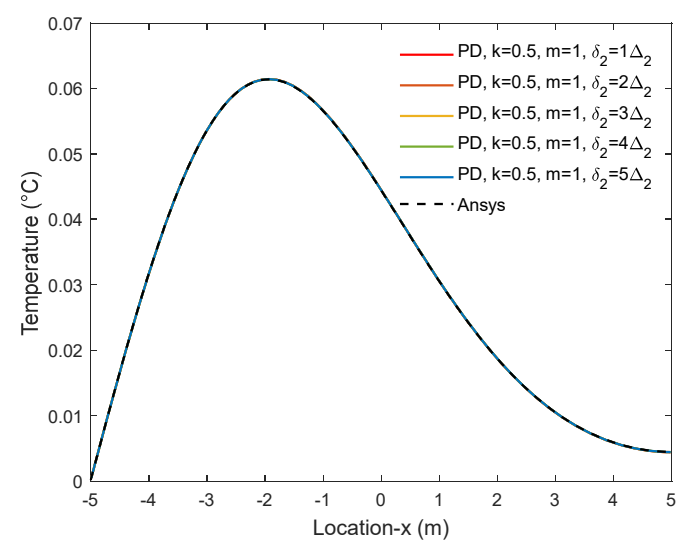

(b)

Figure 15. Temperature variations from PD and FEM predictions when (a) $t=3 s,(b) t=6 s$ at $\mathrm{y}=0, \mathrm{k}=0.5, \mathrm{~m}=1$.

As can be observed from Fig. 15, results from PD and FEM predictions for various horizons are in a good agreement.

\subsubsection{Plate with Non-uniform Discretisation and Different Horizon Sizes}

The fourth case inherits the same discretisation configuration with the third case, i.e. $\Delta_{1}=$ $0.02 \mathrm{~m}, \Delta_{2}=0.04 \mathrm{~m}$. However, the horizon size in Region 1 reduces to half size of the horizon size in Region 2, i.e. $\delta_{2}=2 \delta_{1}$. Since both $\mathrm{k}$ and $\mathrm{m}$ parameters are equal to 0.5 , each horizon has identical number of material points. Same time step size $5.0 \times 10^{-4} \mathrm{~s}$ is adopted to obtain the temperature variations along the horizontal central axis when time $t=3 \mathrm{~s}$ and $\mathrm{t}=6 \mathrm{~s}$.

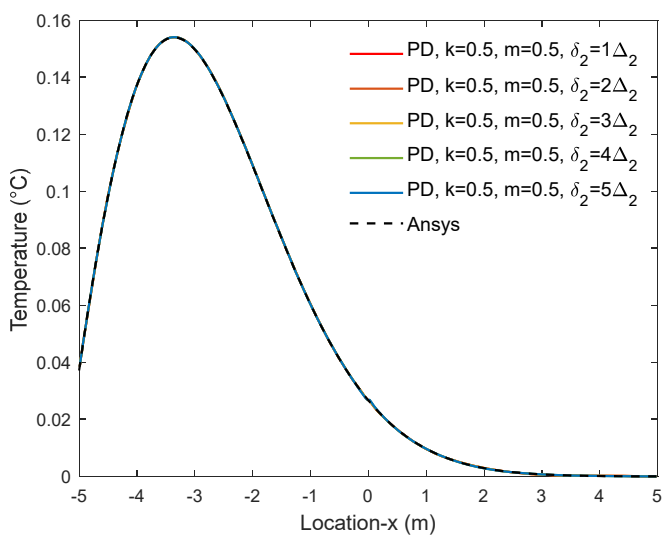

(a)

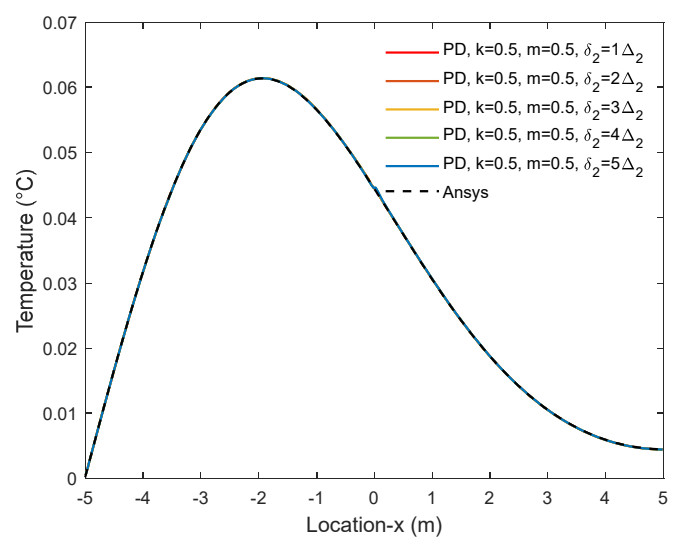

(b)

Figure 16. Temperature variations from $P D$ and FEM predictions when (a) $t=3 s,(b) t=6 s$ at $\mathrm{y}=0, \mathrm{k}=0.5, \mathrm{~m}=0.5$. 
The PD results mostly agree with FEM results for various horizon sizes (see Fig. 16). However, with the horizon size increases in each region, a slight difference is observed at the interface of Regions 1 and 2 for both $\mathrm{t}=3 \mathrm{~s}$ and $\mathrm{t}=6 \mathrm{~s}$.

\subsection{Square plate with an insulated crack}

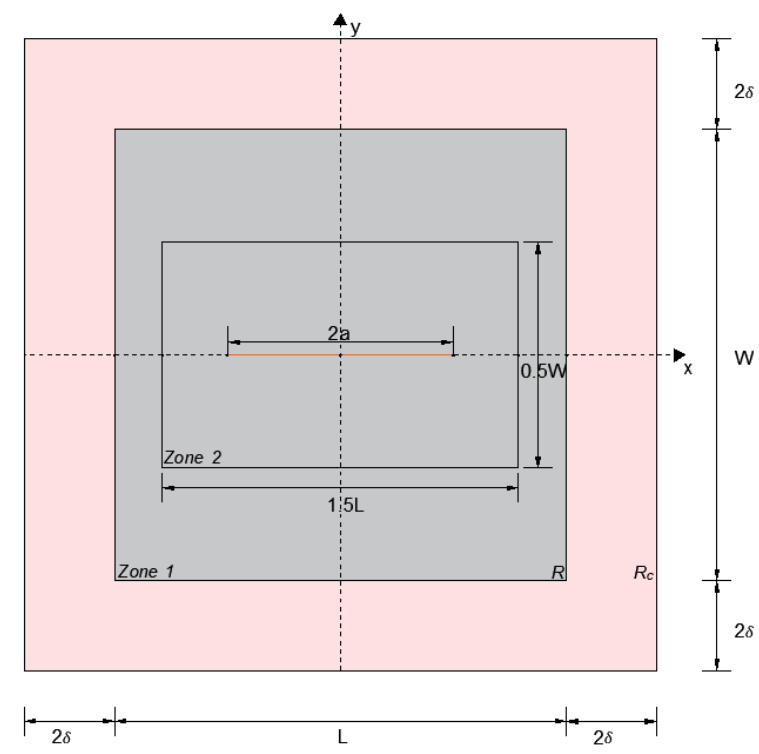

Figure 17. Peridynamic model of the plate with an insulated crack

For the final example problem as shown in Fig. 17, the isotropic square plate is considered with $2 \mathrm{~cm}$ in length (L), $2 \mathrm{~cm}$ in width (W) and $0.01 \mathrm{~cm}$ in thickness $(h)$. The plate has an insulated crack in the middle with a crack length of $2 \mathrm{a}=1 \mathrm{~cm}$. The specific heat capacity $C_{v}$, thermal conductivity $k$ and mass density $\rho$ are specified as $C_{v}=1 \mathrm{~J} / \mathrm{kgK}, k=1.14 \mathrm{~W} / \mathrm{cmK}$ and $\rho$ $=1 \mathrm{~kg} / \mathrm{cm}^{3}$, respectively. The plate is subjected to the following initial and boundary conditions:

$$
\Theta(x, y, z, t=0)=0^{\circ} \mathrm{C} \quad-\frac{L}{2} \leq x \leq \frac{L}{2}, \quad-\frac{W}{2} \leq y \leq \frac{W}{2}
$$

and

$$
\begin{gathered}
\Theta\left(x, \frac{W}{2}, t\right)=100^{\circ} \mathrm{C}, \quad \Theta\left(x,-\frac{W}{2}, t\right)=-100^{\circ} \mathrm{C}, \quad \mathrm{t}>0 \\
\Theta, x\left(\frac{L}{2}, y, t\right)=0^{\circ} \mathrm{C}, \quad \Theta, x\left(-\frac{L}{2}, y, t\right)=0^{\circ} \mathrm{C}, \quad \mathrm{t}>0
\end{gathered}
$$

Since the there is an insulated crack in the geometry, in order to evaluate the effect of dual horizon concept and non-uniform discretisation, the geometry is split into two regions in PD model as in the previous two numerical examples. Note that the definition of regions (zones) in this numerical example is different than the previous two numerical examples. Moreover, $\mathrm{k}$ and $\mathrm{m}$ parameters are defined similarly as in the previous two examples. 


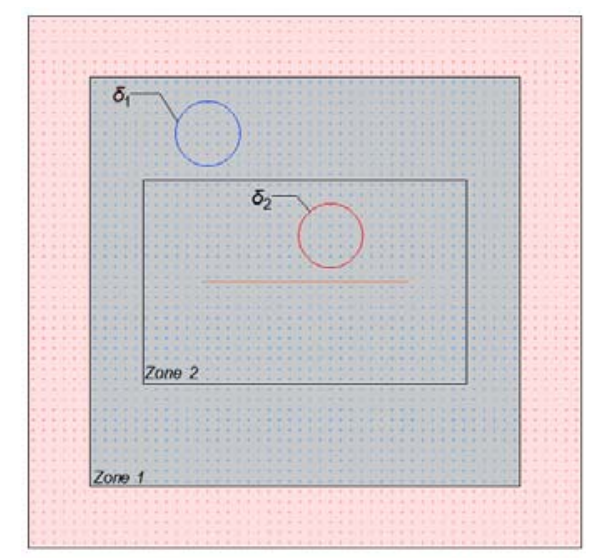

Figure 18. PD model with uniform discretisation and identical horizon sizes

As shown in Fig. 18, in this initial case the plate is uniformly discretised $(\mathrm{k}=1)$ with $0.01 \mathrm{~cm}$ spacing and the same horizon size is used in both zones $(\mathrm{m}=1)$. The peridynamic solution of the temperature variations along the vertical central axis is evaluated with a time step size of $1.0 \times 10^{-5} \mathrm{~s}$ for various horizon sizes and compared with FEM results.

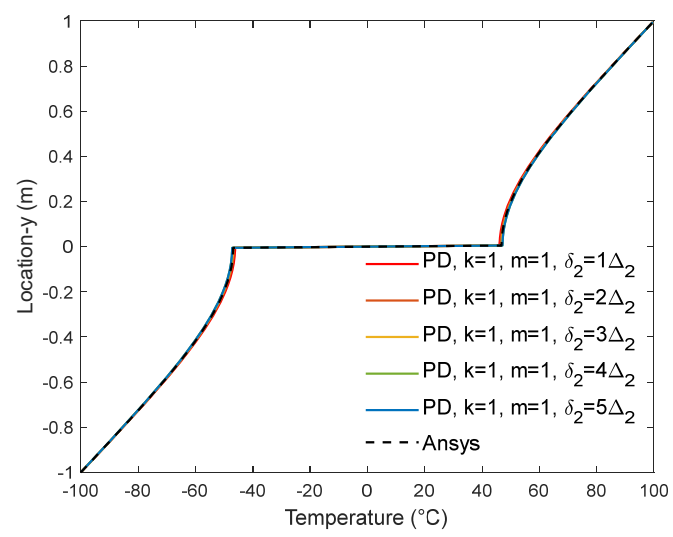

Figure 19. Temperature variations from $P D$ and FEA predictions at $x=0$ when $k=1, m=1$

As can be seen in Fig. 19, the numerical results from PD analyses for various horizon sizes with uniform discretisation agree well with FEM results. 


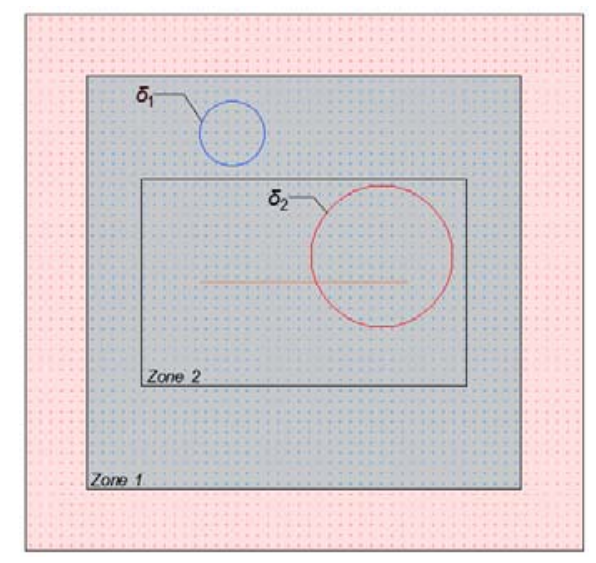

Figure 20. PD model with uniform discretisation and different horizon sizes

To investigate the dual horizon size concept, this second case (see Fig. 20) utilises uniform discretisation as in the first case $\left(\mathrm{k}=1, \Delta_{1}=0.01 \mathrm{~cm}, \Delta_{2}=0.01 \mathrm{~cm}\right)$ and the horizon size in Region 2 is doubled due to the existence of insulated crack in this region $\left(m=0.5, \delta_{2}=2 \delta_{1}\right)$. This case adopts the same time step size as the first case.

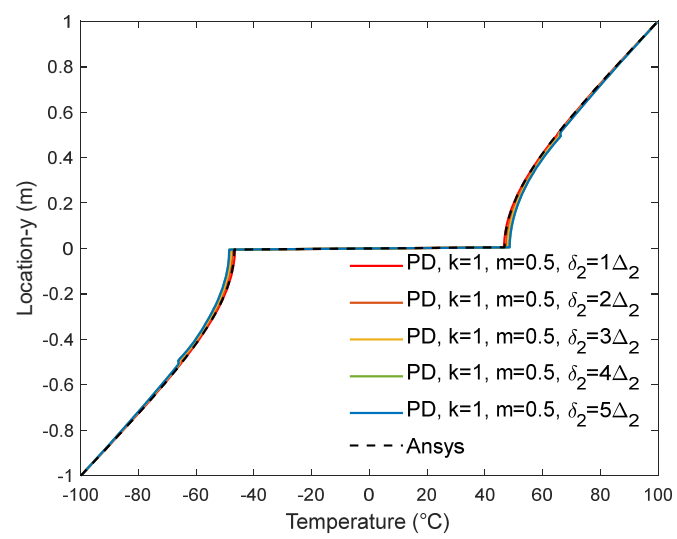

Figure 21. Temperature variations from $P D$ and FEA predictions at $\mathrm{x}=0$ when $\mathrm{k}=1, \mathrm{~m}=0.5$

As shown in Fig. 21, there is a good agreement between PD and FEM results obtained along the vertical central axis. However, as the horizon size increases, a slight difference is observed at the interface between Region 1 and Region 2. 


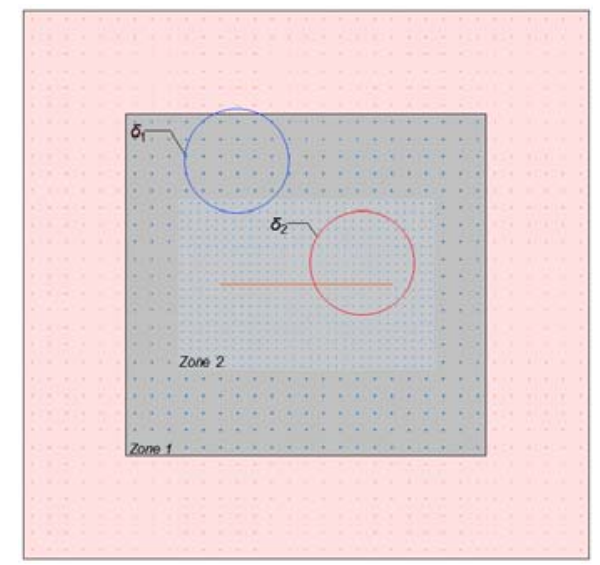

Figure 22. PD model with non-uniform discretisation and identical horizon sizes

Since coarse mesh requires less computational time, in this third case, the spacing in Region 1 is increased by two times compared with the first case $\left(\mathrm{k}=2, \Delta_{1}=0.02 \mathrm{~cm}, \Delta_{2}=0.01 \mathrm{~cm}\right)$. To evaluate the effect of non-uniform discretization, this third case (see Fig. 22) utilises same horizon sizes in all regions $\left(m=1, \delta_{2}=\delta_{1}\right)$. The time step size is the same as the first case.

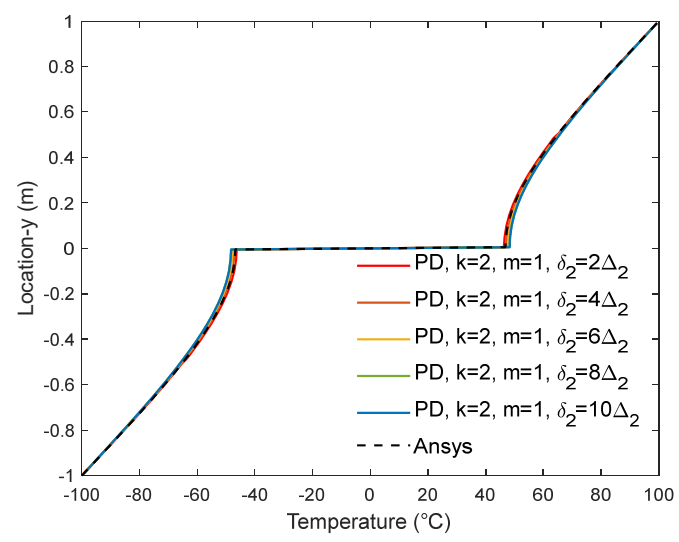

Figure 23. Temperature variations from PD and FEM predictions at $\mathrm{x}=0$ when $\mathrm{k}=2, \mathrm{~m}=1$

As can be observed in Fig. 23, the PD predictions for the temperature variations along the vertical central axis agree well with the FEM results for various horizon sizes. 


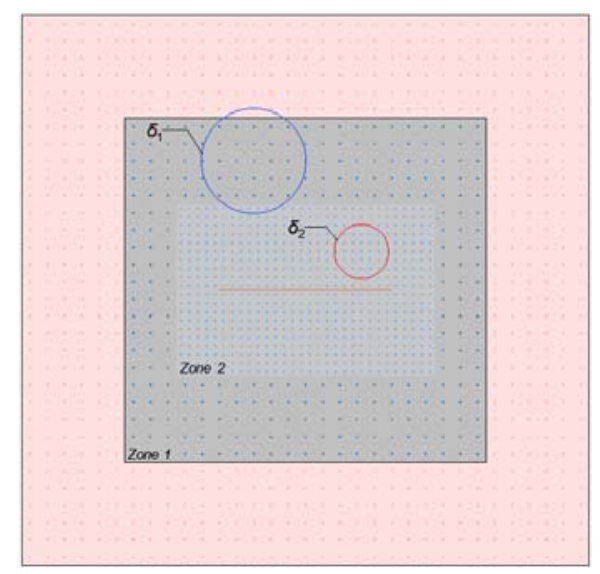

Figure 24. PD model with non-uniform discretisation and different horizon sizes

If horizon contains more material points, the computational time will increase. Therefore, in the last case, horizon size in Region 2 is reduced to half size compared with the horizon size in Region 1 ( $\left.m=2, \delta_{1}=2 \delta_{2}\right)$ as shown in Fig. 24. The discretisation size is the same as in the third case $\left(\mathrm{k}=2, \Delta_{1}=0.02 \mathrm{~cm}, \Delta_{2}=0.01 \mathrm{~cm}\right)$. Since both mesh ratio, $\mathrm{k}$ and horizon size ratio, $\mathrm{m}$ have values of 2 , horizons in Regions 1 and 2 contain identical number of material points.

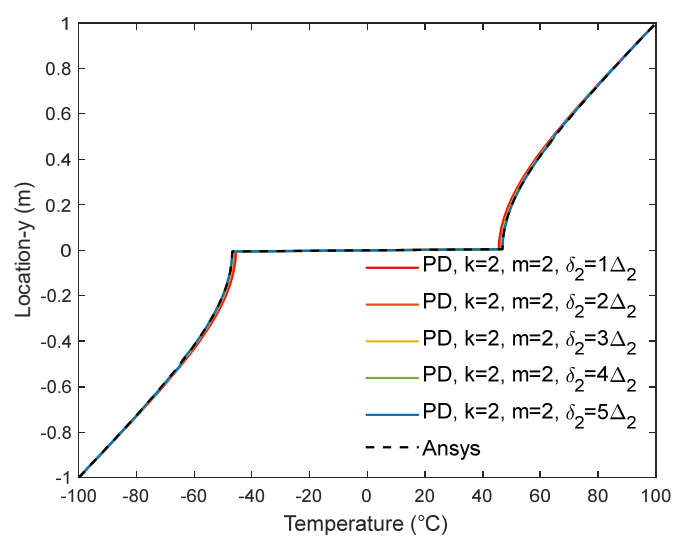

Figure 25. Temperature variations from $P D$ and FEM predictions at $x=0$ when $k=2, m=2$

As shown in Fig. 25, the PD temperature predictions obtained along the vertical central axis agree well with FEM results. As the horizon size increases, a small difference is observed at the interface between Regions 1 and 2.

\subsection{Femtosecond laser heating of a metal film}

In order to demonstrate the applicability of the present formulation for nano-scale structures, femtosecond laser heating of a metal film problem is considered as shown in Fig. 26. The thin metal film is $20 \mathrm{~nm}$ in length, $20 \mathrm{~nm}$ in width and is subjected to femtosecond laser heating for 
a short period time $t=1 p s$. Its specific heat capacity and thermal conductivity are specified as $c=2.5 \times 10^{6} \mathrm{~J} / \mathrm{m}^{3} \mathrm{~K}, k=315 \mathrm{~W} / \mathrm{mK}$, respectively. The metal film has a discretization size of $\Delta x=L / 100 \mathrm{~nm}$ in $x, y$ directions respectively, and a time step size of $\Delta t=2 \times 10^{5} p s$ is adapted in the numerical model.

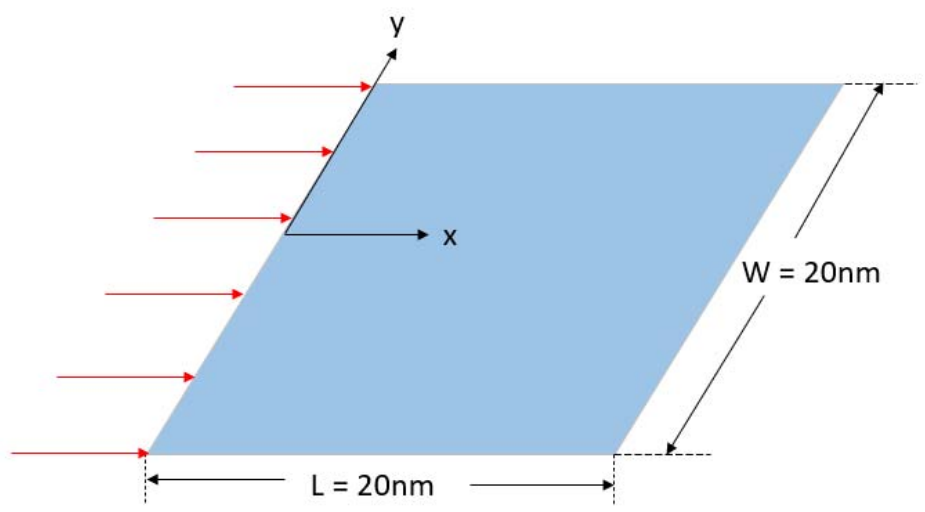

Figure 26. A thin metal film subjects an ultra-short pulse laser

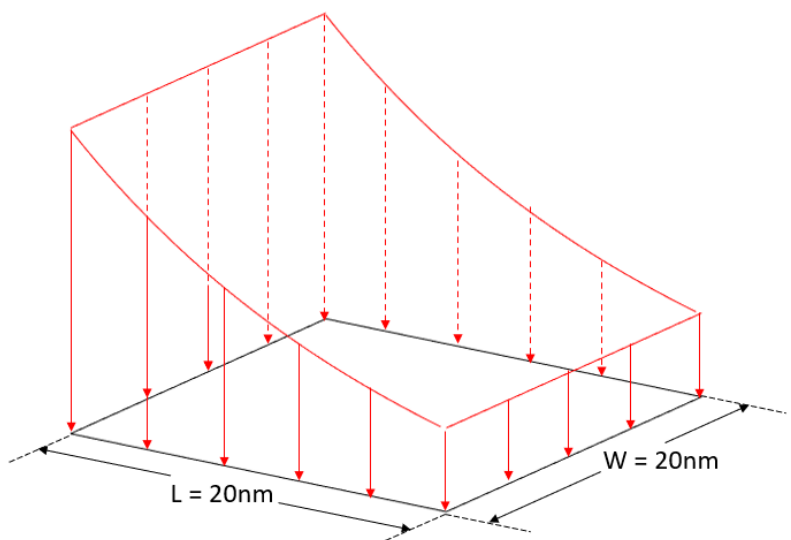

Figure 27. Ultra-short pulse laser source heat capacity distribution on the metal film Initial conditions are given by

$$
\begin{aligned}
& T(x, y, t=0)=0{ }^{\circ} \mathrm{C}, \\
& \frac{\partial T(x, y, t=0)}{\partial t}=0,
\end{aligned}
$$

and boundary conditions are given by

$$
\begin{aligned}
& \frac{\partial T(x=0, y, t)}{\partial x}=0, \\
& \frac{\partial T(x=L, y, t)}{\partial x}=0 .
\end{aligned}
$$

The femtosecond laser heating in the numerical model was approximated by considering a heat source in the form of a Gaussian profile (see Fig. 27) which is given by [15] 


$$
s(x, y, t)=0.94 J_{0}\left(\frac{1-R}{t_{0} l}\right) e^{-\frac{x}{l}-\frac{a\left|t-2 t_{0}\right|}{t_{0}}},
$$

where the laser fluence is $J_{0}=13.4 \mathrm{~J} / \mathrm{m}^{2}$, the reflectivity is $R=0.93$, the radiation penetration depth is $l=15.3 \times 10^{-9} \mathrm{~m}$, the full-width-at-half maximum duration of the laser pulse is $t_{0}=100 \times 10^{-15} \mathrm{~s}$, and the Gaussian parameter is $a=1.992$.

In order to validate the PD model, the temperature history at $x=0, y=0$ has been obtained and compared with the experimental data [15]. The temperature $T_{n}$ is normalized by

$$
T_{n}=\frac{T}{T_{m}}
$$

where $T_{m}$ is the maximum value of the temperature during $1 p s$ period. As can be observed from the Fig.28 that the PD thermal diffusion model has a close agreement with the experimental data.

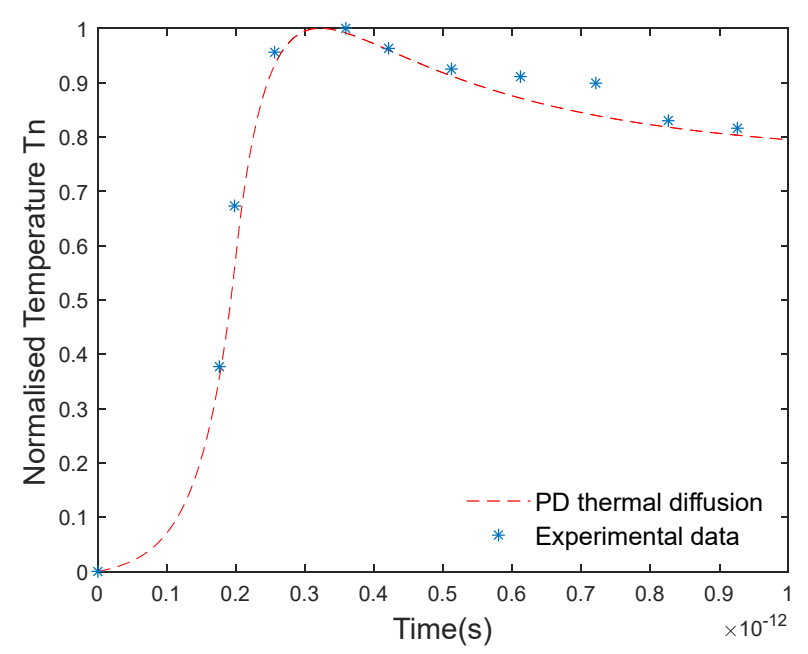

Figure 28. Temperature variations at $x=0, y=0$

\subsection{Plate subjected to a thermal shock loading}

For the final numerical example, a square plate subjected to thermal shock loading is considered as shown in Fig. 28. The plate is made of an isotropic material with insulated boundaries. The geometric parameters are $10 \mathrm{~m}$ in length $L$ and width $W$ with $1 \mathrm{~m}$ thickness $H$.The specific heat capacity $c_{v}$, thermal conductivity $k$ and mass density $\rho$ are specified as $c_{v}=1 \mathrm{~J} / \mathrm{m}^{3} \mathrm{~K}, k=1 \mathrm{~W} / \mathrm{mK}$ and $1 \mathrm{~kg} / \mathrm{m}^{3}$ respectively. The initial conditions and boundary conditions are stated as

$$
\begin{gathered}
\Theta(x, y>0, t=0)=0{ }^{\circ} \mathrm{C} \\
\Theta(x, y<0, t=0)=0.5^{\circ} \mathrm{C}
\end{gathered}
$$

and

$$
\Theta, x(x=5, y, t)=0^{\circ} \mathrm{C},
$$




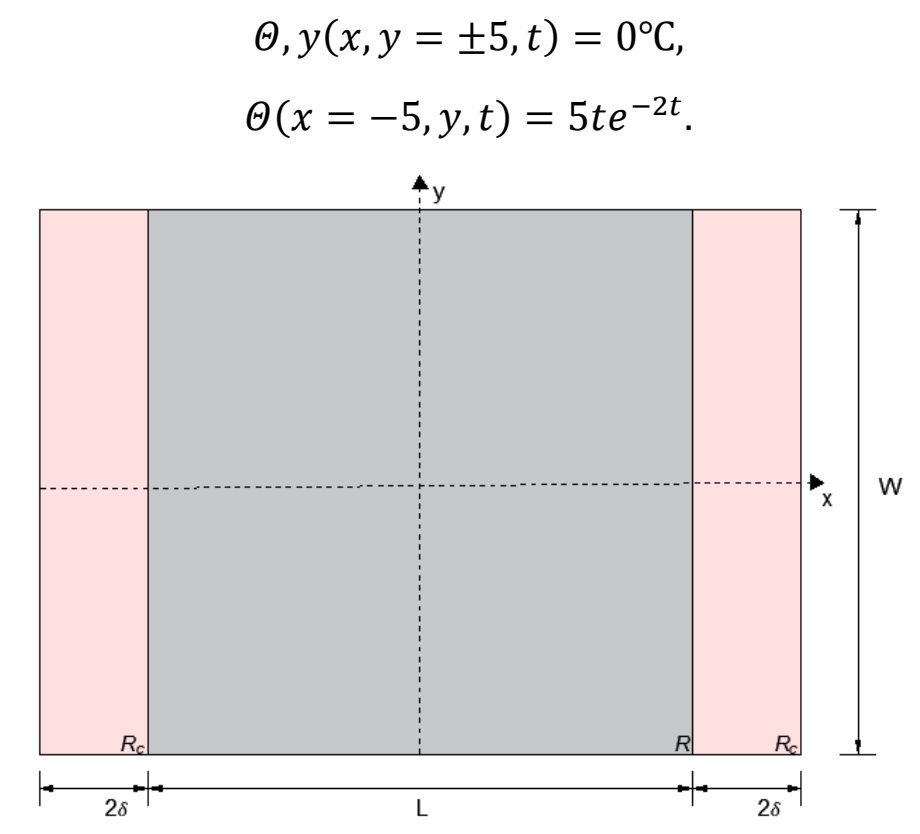

Figure 28. Numerical model of the plate under thermal shock loading

In the peridynamic model, the spacing between material points is $\Delta x=L / 400$ and a step size of $\Delta t=5 \times 10^{-4} s$ is used.

Peridynamic results are compared with the results from a FEM model for verification purposes. The temperature distribution of the plate at $t=3 \mathrm{~s}$ in PD model and FEM mode are depicted in the Fig. 29. Moreover, the temperature variation along $x, y= \pm 5 m$ and $x, y= \pm 2.5 m$ are shown in Fig. 30. As can be seen in these figures, the temperature distribution in PD model has a good agreement with the FEM model.

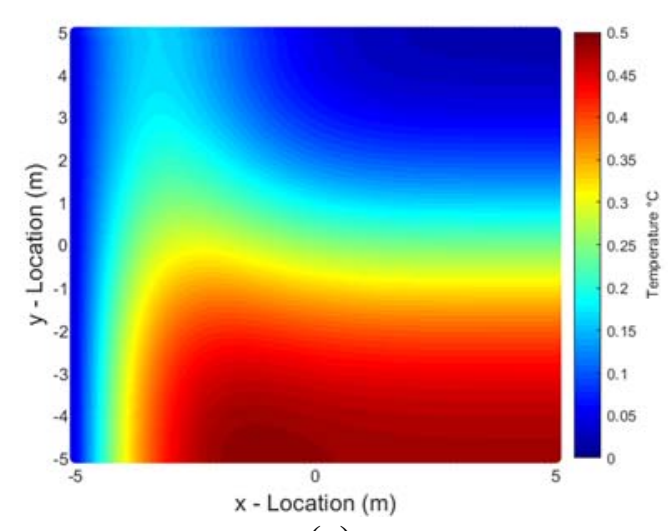

(a)

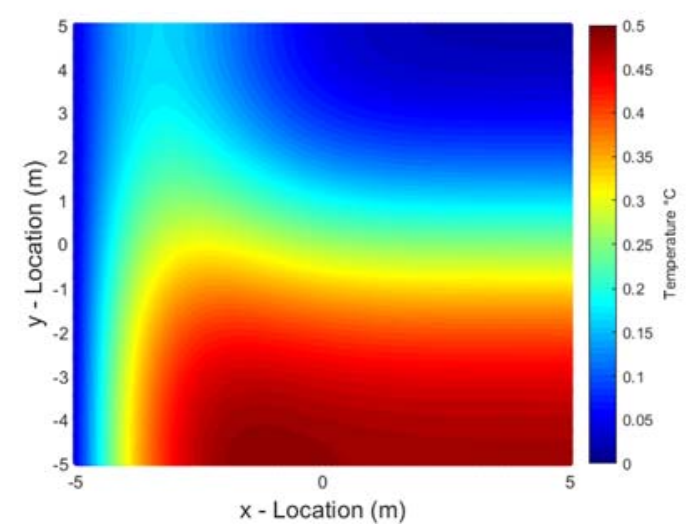

(b)

Figure 29. Temperature distribution on the plate at $t=3 \mathrm{~s}$ (a) PD, (b) FEM 


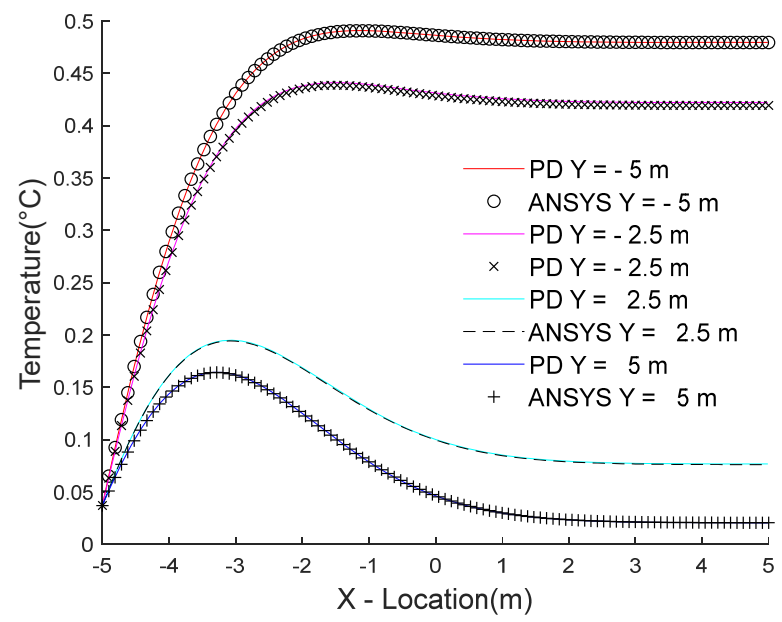

Figure 30. Temperature variation along $x, y= \pm 5 \mathrm{~m}$ and $x, y= \pm 2.5 \mathrm{~m}$

\section{Conclusions}

In this study, Dual Horizon Peridynamic formulation is presented for thermal diffusion analysis. Dual Horizon Peridynamics allows utilisation of variable discretisation and horizon size inside the computational domain which can bring significant advantages to peridynamic simulations in terms of computational time. To demonstrate the capability of the Dual Horizon Peridynamics formulation, three different example problems are considered including a square plate with temperature and no flux boundary conditions, a square plate under thermal shock loading, and a square plate with an insulated crack. For each of these problems, the solution domain is divided into two regions which can have different discretisation and horizon sizes. Five different horizon sizes were considered to see the effect of the horizon size. For all the problems that were considered, good agreement was obtained between PD predictions and FEM results. If different discretisation size or horizon size is utilised in different regions, a slight difference can be observed between PD and FEM results at the interface between the two regions and the difference increases as the horizons size increases. Hence, it can be concluded that presented Dual Horizon Peridynamic analysis can be effectively utilised for problems with different discretisation and horizon sizes.

\section{References}

1. Silling, S.A., 2000. Reformulation of elasticity theory for discontinuities and long-range forces. Journal of the Mechanics and Physics of Solids, 48(1), pp.175-209.

2. Oterkus, S., Madenci, E. and Agwai, A., 2014. Fully coupled peridynamic thermomechanics. Journal of the Mechanics and Physics of Solids, 64, pp.1-23.

3. Oterkus, S., Madenci, E. and Oterkus, E., 2017. Fully coupled poroelastic peridynamic formulation for fluid-filled fractures. Engineering geology, 225, pp.19-28. 
4. Diyaroglu, C., Oterkus, S., Oterkus, E., Madenci, E., Han, S. and Hwang, Y., 2017.

Peridynamic wetness approach for moisture concentration analysis in electronic packages.

Microelectronics Reliability, 70, pp.103-111.

5. Gerstle, W., Silling, S., Read, D., Tewary, V. and Lehoucq, R., 2008. Peridynamic simulation of electromigration. Comput Mater Continua, 8(2), pp.75-92.

6. Bobaru, F. and Duangpanya, M., 2012. A peridynamic formulation for transient heat conduction in bodies with evolving discontinuities. Journal of Computational Physics, 231(7), pp.2764-2785.

7. Oterkus, S., Madenci, E. and Agwai, A. (2014). Peridynamic thermal diffusion. Journal of Computational Physics, 265, pp.71-96.

8. Oterkus, S., Madenci, E., Oterkus, E., Hwang, Y., Bae, J. and Han, S., 2014, May. Hygrothermo-mechanical analysis and failure prediction in electronic packages by using peridynamics. In 2014 IEEE 64th Electronic Components and Technology Conference (ECTC) (pp. 973-982). IEEE.

9. Xue, T., Zhang, X. and Tamma, K.K., 2018. A two-field state-based peridynamic theory for thermal contact problems. Journal of Computational Physics, 374, pp.1180-1195.

10. Wang, L.J., Xu, J.F. and Wang, J.X., 2016. The Green's functions for peridynamic nonlocal diffusion. Proceedings of the Royal Society A: Mathematical, Physical and Engineering Sciences, 472(2193), p.20160185.

11. Liao, Y., Liu, L., Liu, Q., Lai, X., Assefa, M. and Liu, J., 2017. Peridynamic simulation of transient heat conduction problems in functionally gradient materials with cracks. Journal of Thermal Stresses, 40(12), pp.1484-1501.

12. Madenci, E. and Oterkus, E., 2014. Peridynamic theory and its applications (Vol. 17). New York: Springer.

13. Ren, H., Zhuang, X., Cai, Y. and Rabczuk, T., 2016. Dual-horizon peridynamics. International Journal for Numerical Methods in Engineering, 108(12), pp.1451-1476.

14. Ren, H., Zhuang, X. and Rabczuk, T., 2017. Dual-horizon peridynamics: A stable solution to varying horizons. Computer Methods in Applied Mechanics and Engineering, 318, pp.762-782.

15. Qiu, T.Q., Juhasz, T., Suarez, C., Bron, W.E. and Tien, C.L., 1994. Femtosecond laser heating of multi-layer metals-II. Experiments. International Journal of Heat and Mass Transfer, 37(17), pp.2799-2808. 